physical dependence varies, the most important factor being the degree to which function of the central nervous system is altered by the drug. ${ }^{4}$

Our patient received a substantial quantity of powerful drugs acting on the central nervous system over a fairly short period to combat severe pain. In the postoperative period there was no pain and all systemic analgesics were therefore stopped. She then developed the withdrawal syndrome.

Normally after a major operation patients require opiate analgesics to relieve pain. Opiate withdrawal symptoms, consequent on preoperative iatrogenic preconditioning, are therefore highly unlikely in the early postoperative stage. Gradually as postoperative pain stops the analgesics are reduced, and this permits some weaning from the narcotic. In this case the morphine acted more effectively in one part of the body (the spinal cord) after the operation than it had done before the operation and yet less effectively in another part, causing withdrawal symptoms. It was this that was so disconcerting and unexpected. Indeed, the diagnosis was made only retrospectively after her complete recovery, coinciding with negative results of all laboratory investigations.

As the use of intrathecal morphine increases more patients may develop withdrawal symptoms in the postoperative period, and doctors should therefore be warned of this possibility. Since opiate withdrawal symptoms are easily relieved it is to the patient's immediate benefit that the diagnosis is made early and appropriate treatment begun.

${ }^{1}$ Mathews ET, Abrams LD. Intrathecal morphine in open heart surgery. Lancet 1980 ;ii :543.

${ }^{2}$ Gjessing J, Tomlin PJ. Postoperative pain control with intrathecal morphine. Anaesthesia $1981 ; 36: 268-76$

${ }^{3}$ Tung AS, Tenicela R, Winter PM. Opiate withdrawal syndrome following intrathecal administration of morphine. Anesthesiology 1980;53: 340.

${ }^{4}$ Jerome $\mathrm{H}$ Jaffe. Drug addiction and drug abuse. In: Goodman LS, Gilman A, eds. The pharmacological basis of Therapeutics. 6th ed. New York: Macmillan Publishing Co Inc, 1980: 535-84.

(Accepted 19 May 1981)

University Department of Anaesthetics, Queen Elizabeth Hospital Birmingham B15 2TH

F M MESSAHEL, FFARCS, senior registrar

P J TOMLIN, FFARCS, senior lecturer

\section{False-negative results of Hemoccult test in colorectal cancer}

Testing for faecal occult blood using the Hemoccult test (Smith Kline Instrument $\mathrm{Co}$ ) has been advocated for screening for colorectal cancer in populations at risk, ${ }^{1}$ and its use is suggested for detecting early recurrence after resection of cancers of the colon and rectum. False-negative reactions occur with the Hemoccult test ${ }^{2}$ in patients with proved carcinoma of the large bowel and rectum.

In our series of patients the false-negative results of tests for occult blood corresponded to the lack of surface ulceration of the tumour.

\section{Patients, methods, and results}

Twenty-eight patients with histologically proved carcinoma of the colon and rectum were studied prospectively. Before bowel preparation for operation was started three consecutive stools from each patient were each tested in two separate places using the Hemoccult cards as recommended by Greegor $^{1}$ (in four patients only two consecutive stool specimens were obtained for testing). The patients were not placed on a meat-free diet since this restriction is unnecessary according to the manufacturer's instruction leaflet. No patient received vitamin C supplements, a recognised cause of false-negative reactions. ${ }^{2}$ As each test was read at 30 seconds, there were no false-negative results related to the storage of specimens. ${ }^{3}$ Weak positive reactions were classified as positive for faecal occult blood.
Results of Hemoccult test for faecal occult blood in 28 patients with histologically proved carcinoma of the colon and rectum

\begin{tabular}{cccc}
\hline $\begin{array}{c}\text { No of positive results } \\
\text { (out of 6) }\end{array}$ & No of patients & $\begin{array}{c}\text { No of patients with } \\
\text { ulcerated tumour }\end{array}$ & $\begin{array}{c}\text { No of patients with } \\
\text { non-ulcerated tumour }\end{array}$ \\
\hline 0 & 5 & 0 & 5 \\
1 & 1 & 0 & 1 \\
2 & 4 & 4 & 0 \\
4 & 7 & 5 & 2 \\
6 & 11 & 11 & 0 \\
\hline
\end{tabular}

Each patient underwent resection of the tumour, and the presence or absence of ulceration of the tumour was recorded after examination of the resected specimen.

Occult blood was found in the stools of 23 patients, of whom 21 had ulcerated tumours (see table). Five patients failed to yield any positive results on tests for faecal occult blood despite having a proved carcinoma; all of these patients had non-ulcerated Dukes B tumours-that is, local spread through the bowel wall but no regional lymph node metastases.

\section{Comment}

Carcinomas of the large bowel and rectum are known to shed blood, which can be detected in the faeces. The initial experience with the guaiac resin test for faecal occult blood led to a reduction in sensitivity because of the high numbers of false-positive reactions. ${ }^{1}$ The manufacturers claim, however, that the Hemoccult test has detected blood in vitro in a dilution of $1 / 5000$; this should detect a blood loss of $0.04 \mathrm{ml} / 24$ hours in the average stool of $75-200 \mathrm{~g}$ (UK figures). Heinrich, ${ }^{4}$ however, reported false-negative reactions with the Hemoccult with measured faecal blood losses of $25 \mathrm{ml} / 24$ hours for reasons not yet fully understood. An increasing number of falsenegative reactions using the Hemoccult tests with decreasing size of tumour as measured by the mean area was reported by Songster $e t$ al, ${ }^{5}$ but they made no comment about the surface characteristics of the lesion.

Our study indicates that ulceration of the tumour is an important factor in the false-negative hemoccult reaction. It is logical to presume that non-ulcerated tumours are less likely to bleed into the lumen of the bowel, and hence a negative result of a test for faecal occult blood would result despite the presence of a carcinoma.

Serial Hemoccult testing is widely used as a screening test for colorectal cancer. The results of our preliminary study suggest that an appreciable number of non-ulcerated tumours would be missed.

${ }^{1}$ Greegor DH. Diagnosis of large bowel cancer in the asymptomatic patient. F AMA 1967;201:943-5.

2 Jaffec RM, Kaston B, Young MD, MacLowry N. False negative stool occult blood tests caused by ingestion of ascorbic acid (vitamin C). Ann Intern Med $1974 ; 83: 824-6$.

${ }^{3}$ Fleisher M, Schwartz MK, Winawer SJ. The false negative haemoccult test. Gastroenterology 1977;72:782-4.

${ }^{4}$ Heinrich HC. Occult blood tests. Lancet 1980;i:822-3.

${ }^{5}$ Songster CL, Barrows GH, Jarrett DD. Immunochemical detection of faecal occult blood. Cancer 1980;45:1099-102.

(Accepted 20 May 1981)

Western General Hospital, Edinburgh EH4 2XU

C D M GRIFFITH, FRCSED, surgical registrar, gastrointestinal unit D J TURNER, $\mathrm{MB}, \mathrm{CHB}$, surgical senior house officer, general surgical unit J H SAUNDERS, FRCSGLAS, consultant surgeon, gastrointestinal unit

\section{Correction}

Anaphylactic reaction to low-molecular-weight porcine factor VIII concentrates

An error occurred in this paper by Drs J G Erskine and J F Davidson (20 June, p 2011). In lines 8 and 9 of the case report "an intramuscular injection of $10 \mathrm{mg}$ chlorpheniramine maleate" should have read: "an intravenous injection ..." 